\title{
A search for new magnetic stars in stellar groups and open clusters
}

\author{
D. Kudryavtsev ${ }^{1}$, I. Romanuyk ${ }^{1}$, \\ and V. Elkin ${ }^{2}$ \\ ${ }^{1}$ Special Astrophysical Observatory of the RAS, Nizhny Arkhyz, Russia \\ email: dkudr@sao.ru, roman@sao.ru \\ ${ }^{2}$ Center for Astrophysics, University of Central Lancashire, Preston, UK, \\ email: vgelk@sao.ru
}

\begin{abstract}
We present new results of our ongoing project to find new magnetic stars in stellar groups and open clusters. Observations are carried out at the $6-\mathrm{m}$ and $1-\mathrm{m}$ telescopes of the Special Astrophysical Observatory. Candidates are selected by analyzing the profiles of the $\lambda 5200$ broad, continuum features with low resolution spectra (a modification of the Cramer $\&$ Maeder method). These candidates are observed then with high resolution and a Zeeman analyzer. We present the measurements of 6 new magnetic stars and discuss results for 2 stars with outstanding magnetic fields. To date we found 31 new magnetic stars, listed for the most part in catalogues of stellar groups. Several stars have very strong magnetic fields, among them is the $\mathrm{SrCrEu}$ star HD 178892 with a surface magnetic field $\mathrm{B}_{\mathrm{s}} \geqslant 20 \mathrm{kG}$.
\end{abstract}

Keywords. Stars: chemically peculiar, stars: magnetic fields, Galaxy: open clusters, stars: individual; (HD 178892)

\section{Introduction}

At present we know about 250 magnetic CP stars (Romanyuk 2000), that is about $3 \%$ of the total number of known CP stars (Renson et al. 1991). Such a small percentage reflects that investigations of magnetic fields may be made only with large telescopes, where observing time is in high demand. Before the appearance of CCD detectors it was possible to observe stars as faint as $V=8$ with large telescopes. Now we can go two magnitudes fainter. This permits us to make a comparative analysis of the characteristics of magnetic stars relative to Galactic structure in the solar neighborhood.

We are observing stars in open clusters and in the solar neighborhood. Investigations of nearby stars attracted our attention as a result of the statistical analysis of the spatial distribution and motions of magnetic CP stars (Kudryavtsev \& Romanyuk 2003). Romanyuk \& Kudryavtsev (2001) had suggested some primary orientations of the magnetic fields of such stars. However these conclusions were based on insufficient data.

Starting in 2000 we searched for new magnetic CP stars using the catalogues of Egret \& Jaschek (1981), Renson (1992), Kopylov (1987), and Niedzielski \& Muciek (1988). Our first list from the catalogue of CP stars in stellar groups (Egret \& Jaschek 1981) had only 3 stars from open clusters. Now we are trying to expand the list of candidates by observing stars in open clusters with the SAO 1-m telescope.

\section{Observations and data reduction}

Obtaining Zeeman spectra of many stars would take considerable observing time at the 6 - $m$ telescope. Thus we need a criterion for the probability of finding strong magnetic 
fields. Cramer \& Maeder (1980) showed that the depth of the $\lambda 5200$ broad continuum feature may be as an indicator of the presence of a magnetic field. Our method is a modification of this method, but we use low resolution spectra rather than a photometric index. Stars are first observed with the low resolution spectrograph UAGS on the SAO 1 -m telescope. Then we select stars whose depth of spectral pecularities is $\geqslant 10 \%$ in the $\lambda 5200$ region.

We found magnetic fields by measuring their longitudinal components using Zeeman spectra observed with Main Stellar Spectrograph of the 6-m telescope with circular polarisation analysers (Naidenov \& Chuntonov 1976, Chountonov 2000). The spectra were centered at $\lambda 4500$ with a resolution of about 15000 . The Zeeman shifts in the spectra of magnetic stars are very subtle. Thus we obtained at least three spectra for each star on different dates. We deviated from this rule only when the magnetic field was rather strong and its presence was obvious. Then we sometimes obtained only two measurements. Data reductions were made in ESO MIDAS using the programs for Zeeman spectra reduction (Kudryavtsev 2000).

Table 1. New magnetic stars. Longitudinal magnetic field measurements.

\begin{tabular}{|c|c|c|}
\hline \multirow{2}{*}{\multicolumn{3}{|c|}{$\frac{\text { JD 2450000+ } B_{\mathrm{e}}(\mathrm{G})}{\mathrm{HD} 34163}$}} \\
\hline & & \\
\hline 2191.505 & -170 & 110 \\
\hline 2624.359 & +190 & 90 \\
\hline 2625.363 & -230 & 60 \\
\hline 2626.356 & -620 & 70 \\
\hline 2917.455 & -290 & 120 \\
\hline 2918.434 & -750 & 130 \\
\hline \multicolumn{3}{|c|}{ HD 40759} \\
\hline 2917.581 & +1970 & 320 \\
\hline 2918.545 & +2050 & 250 \\
\hline \multicolumn{3}{|c|}{ HD 49223} \\
\hline 2624.529 & +330 & 150 \\
\hline 2625.536 & +590 & 160 \\
\hline 2626.482 & -120 & 190 \\
\hline & +340 & 260 \\
\hline 3097.280 & +420 & 260 \\
\hline \multicolumn{3}{|c|}{ HD 49713} \\
\hline 2690.338 & +2200 & 540 \\
\hline 3097.261 & -2880 & 350 \\
\hline \multicolumn{3}{|c|}{ HD 182532} \\
\hline 2805.450 & +620 & 110 \\
\hline 2807 & +570 & 80 \\
\hline 2830.495 & -40 & 150 \\
\hline 2831.458 & +40 & 80 \\
\hline \multicolumn{3}{|c|}{ HD 192224} \\
\hline 2835.531 & +390 & 110 \\
\hline 2840.502 & +220 & 210 \\
\hline 3096.579 & -580 & 70 \\
\hline
\end{tabular}

\section{New magnetic stars}

We found 31 new magnetic stars whose measurements for the most part were published in Elkin et al. (2002), Elkin et al. (2003), and Kudryavtsev et al. (2003). The measurements of the longitudinal magnetic fields are shown in Table 1.

Here we present the latest results: 6 new stars whose magnetic fields were detected for the first time.The stars are poorly studied. For HD 40759 Maitzen \& Vogt (1983) found 
$\Delta \mathrm{a}=0.0028$, which suggested a strong magnetic field. HD 49713 was listed in Babcock (1958) as a star with too wide spectral lines for magnetic measurements. Despite this we found a magnetic field of over $2 \mathrm{kG}$. HD 49713 was also noted by Cramer \& Maeder (1980) as a star with a possibly strong field. HD 40759 is a member of OriOB1. All the other stars are included in the catalogue of stellar groups. During the first half of 2004 we observed with the 1-m telescope and have already selected several candidates. Our observations will continue.

In Table 2 we give only the observed magnetic field extrema of our first 31 magnetic stars as the original measurements were published before. Note that in some cases we have only 2 or 3 spectra for a star and thus possibly have not observed the real extrema, but these values still give some idea about the magnetic field strength.

HD 178892, HD 293764, HD 343872, and HD 349321 with very strong magnetic fields where the longitudinal component is $4 \mathrm{kG}$ or more are marked in gray. We are observing them. For HD 178892 and HD 343872 we already have sufficient results to discuss them.

Table 2. Our first 31magnetic stars

\begin{tabular}{llllll}
\hline HD /BD & Mag & $B_{\mathrm{e}}(\mathrm{G})$ & $B_{\text {extr }}(\mathrm{G})$ & $\mathrm{Pec}$ & Cluster \\
\hline HD 6757 & 7.7 & +2170 & +3100 & $\mathrm{CrEuSi}$ & \\
HD 29925 & 8.3 & -1100 & -200 & $\mathrm{Si}$ & \\
HD 34162 & 8.7 & -750 & +190 & $\mathrm{SrCrEu}$ & Ori OB1 \\
HD 38823 & 7.3 & -2490 & +1520 & $\mathrm{SrEu}$ & \\
HD 39658 & 8.8 & -970 & +1350 & $\mathrm{CrEu}$ & \\
HD 40711 & 8.4 & -650 & +330 & $\mathrm{SrCrEu}$ & Ori OB1 \\
HD 40759 & 8.6 & +1970 & +2050 & $\mathrm{CrEu}$ & \\
HD 49223 & 9.0 & -120 & +590 & $\mathrm{SrEu}$ & \\
HD 49713 & 7.3 & -2880 & +2200 & $\mathrm{CrEuSi}$ & \\
HD 115606 & 8.6 & -760 & +680 & $\mathrm{Cr}$ & \\
HD 134793 & 7.5 & -810 & +950 & $\mathrm{SrEuCr}$ & \\
HD 142554 & 9.8 & -770 & +1740 & $\mathrm{CrEu}$ & \\
HD 158450 & 8.6 & -2980 & +810 & $\mathrm{SrCrEu}$ & \\
HD 168796 & 7.9 & -870 & +510 & $\mathrm{SiSrCr}$ & \\
HD 169887 & 9.0 & -2340 & +2020 & $\mathrm{Si}$ & \\
HD 170565 & 9.1 & +1580 & +1960 & $\mathrm{SrCrEu}$ & \\
HD 170973 & 6.4 & -400 & +630 & $\mathrm{SiCrSr}$ & \\
HD 178892 & 8.9 & +1670 & +8490 & $\mathrm{SrCrEu}$ & \\
HD 182532 & 9.3 & -40 & +620 & $\mathrm{CrEu}$ & \\
HD 189963 & 9.9 & -700 & +360 & $\mathrm{SrCrEu}$ & \\
HD 192224 & 8.9 & -580 & +390 & $\mathrm{CrEu}$ & \\
HD 196691 & 8.6 & -1940 & +2290 & $\mathrm{Si}$ & \\
HD 209051 & 8.8 & -3300 & -1040 & $\mathrm{SrCrEu}$ & \\
HD 231054 & 10.0 & +380 & +2530 & $\mathrm{SiSr}$ & \\
HD 293764 & 9.5 & +3590 & +4040 & $\mathrm{SrCrEu}$ & \\
HD 338226 & 9.8 & +440 & +1490 & $\mathrm{Si}$ & \\
HD 343872 & 9.9 & -760 & +4590 & $\mathrm{SrCrEu}$ & \\
HD 349321 & 9.3 & -5560 & +2190 & $\mathrm{Si}$ & \\
BD +17.3622 & 8.8 & +980 & +1600 & $\mathrm{SrCrEu}$ & \\
BD +32.2827 & 9.9 & -770 & +60 & $\mathrm{SrCrEu}$ & \\
BD +35.3616 & 9.5 & -520 & +540 & $\mathrm{SrEu}$ & \\
\hline
\end{tabular}

\section{Stars with strong magnetic fields}

\section{1. $H D 178892$}

Our observations with the $1-\mathrm{m}$ telescope revealed a prominent feature near $\lambda 5150$. With the 6-m telescope we obtained 17 Zeeman spectra for HD 178892. It possesses a strong 
magnetic field whose longitudinal component can be as great as $8 \mathrm{kG}$. Only 4 stars have comparable or stronger magnetic fields. These are HD 37776, HD 215441, HD 175362, and the recently discovered NGC2244-334 (Bagnulo et al. 2004). All are hot $\left(T_{\text {eff }} \geqslant\right.$ $15000 \mathrm{~K}$ ) and have He or Si anomalies, but HD 178892 is a $\mathrm{SrCrEu}$ star, so it is definitely cooler. Among the SrCrEu stars, HD 178892 is now a record holder for magnetic field strength which makes it very interesting. As it is the coolest HD 178892 might be older than the other four stars with the similar magnetic fields. By carefully determining its age (temperature) this star may help to decide whether only young stars have strong magnetic fields.

Using our measurements of the longitudinal magnetic field we found the rotational period of HD $178892 \mathrm{P}=8.27 \pm 0.08$ days. We also tried to determine the period from Hipparcos photometry, but the star did not show sufficient photometrical variability. Using the longitudinal magnetic field curve we find $B_{\mathrm{s}} \geqslant 20 \mathrm{kG}$. Values of $v \sin i$ vary from 20 (instrumental profile) to $45 \mathrm{~km} \mathrm{~s}^{-1}$ depending on the Landé $\mathrm{g}$ factor. This is the direct evidence of maonetic strenothenino

HD178892 JD2452452.217+ $8.27(+-0.08)$

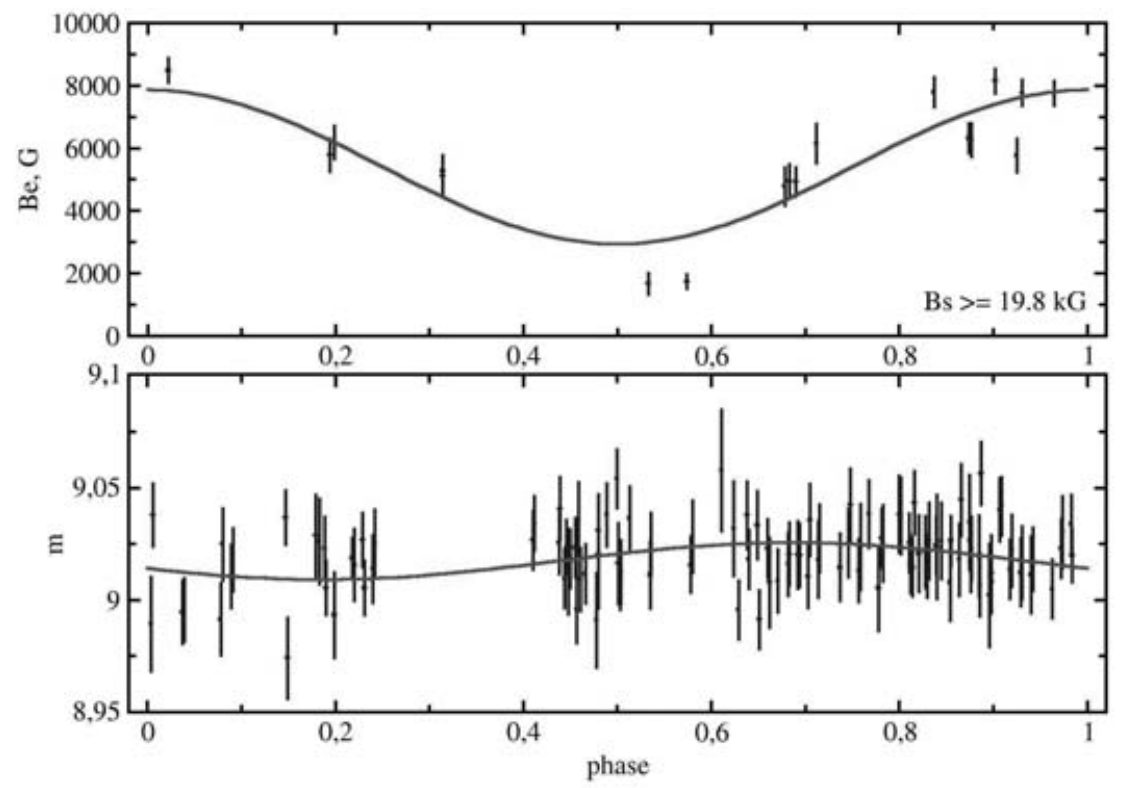

Figure 1. HD 178892, the SrCrEu star with strong magnetic field. The longitudinal magnetic field curve (top) and the HIPPARCOS photometry shown as functions of the rotational period.

\section{2. $H D 343872$}

We have over 20 Zeeman spectra of HD 343872. Using the measurements of the longitudinal magnetic field we found its rotational period was $\mathrm{P}=8.79 \pm 0.02$ days. Tycho photometry did not show any measurable variability. The longitudinal magnetic field curve corresponds with the lower limit of the surface magnetic field $B_{\mathrm{s}} \geqslant 9 \mathrm{kG}$.

With such a surface field $B_{\mathrm{s}}$ magnetic broadening can contribute up to $50 \%$ of the total line width. Values of $v \sin i$ determined by different lines vary from 25 to $35 \mathrm{~km} \mathrm{~s}^{-1}$ depending on the Landé g factor. So the contribution of magnetic broadening cannot be ignored when studying the chemical composition of the stellar atmosphere. 
Preliminary analysis of the Balmer lines profiles shows that HD 343872 has a rather high temperature $(\geqslant 10000 \mathrm{~K}$ ) for a $\mathrm{SrCrEu}$ star and low $\log g$ (about 3.5). Spectroscopic observations by Kroll (1992) resulted in $T_{\text {eff }}=10500 \mathrm{~K}$ and $\log g=3.1$, suggesting that it is an evolved star. In any case the star is rather unusual. It is necessary to further model of its spectra to determine its atmosphere parameters and chemical composition.

\section{References}

Babcock, G. 1958, ApJS 3, 141

Bagnulo, S., Hensberge, H., Landstreet, J.D., Szeifert, T., Wade G.A. 2004, A\&̊A 416, 1149

Cramer, N., Maeder, A. 1980, A\&SAS 41, 111

Chountonov, G.A. 2000, in: Yu.V. Gladolevskij and I.I. Romanyuk (eds.), Magnetic Fields of CP and Related Stars, Proc. of intern. meeting (Moscow), p. 94

Egret, D., Jaschek, M. 1981, Comptes Rendus Symp. Liege, vol. 23, p. 495

Elkin, V.G., Kudryavtsev, D.O., Romanyuk, I.I. 2002, Astron. Letters, 28, 195

Elkin, V.G., Kudryavtsev, D.O., Romanyuk, I.I. 2003, Astron. Letters, 29, 455

Kopylov, I.M. 1987, Astrofizicheskie Issledovaniya (Izv. SAO) 24, 44

Kroll, R. 1992, in: M.M. Dworetsky, F. Castelly, R. Farragiana (eds.), Peculiar Versus Normal Phenomena in A-type and Related Stars, Proc. of IAU Coll. 138 (ASP Conf. Ser.), vol. 44, p. 75

Kudryavtsev, D.O. 2000 in: Yu.V. Gladolevskij and I.I. Romanyuk (eds.), Magnetic Fields of $C P$ and Related Stars, Proc. of intern. meeting (Moscow), p. 84

Kudryavtsev, D.O., Romanyuk, I.I. 2003, Astrophysics 46, 234

Kudryavtsev, D.O., Romanyuk, I.I., Elkin, V.G. 2004, in: Yu.V. Gladolevskij, D.O. Kudryavtsev and I.I. Romanyuk (eds.), Magnetic Stars, Proc. of intern. meeting (Moscow), (in press)

Maitzen, H.M., Vogt, N. 1983, A\&A 123, 48

Naidenov I. D., Chuntonov G. A. 1976, Soobscheniya SAO 16, 63

Niedzielski, A., Muciek, M. 1988, Acta Astronomica 45, 375

Renson, P., Gerbaldi, M., Catalano, F. 1991, A\&AS 89, 429

Renson P. 1992, Bull. Inf. Centre Donnees Stellaires 40, 97

Romanyuk, I.I. 2000 in: Yu.V. Gladolevskij and I.I. Romanyuk (eds.), Magnetic Fields of CP and Related Stars, Proc. of intern. meeting (Moscow), p. 18

Romanyuk I. I., Kudryavtsev D. O. 2001, ASP Conf. Ser. 248, 299 